\title{
Application of Modified Gastric Tube Placement in Patients with Indwelling Gastric Tube
}

\author{
Hua Tang* \\ Jianhu People's Hospital, Yancheng 224700, Jiangsu Province, China
}

*Corresponding author: Hua Tang, 347542321@qq.com

\begin{abstract}
Objective: To explore the effect of modified nasogastric tube placement and Rehabilitation New Liquid Spray in patients with indwelling gastric tube. Methods: Eighty-six cases with indwelling gastric tube in our hospital from January 2020 to May 2021 were randomly selected and divided into the reference group and the research group. The reference group was treated by modified gastric tube placement, and the research group was given the Rehabilitation New Liquid Spray intervention. The effect of the interventions on two groups was observed. Results: The incidence of nasal mucosal injury in the research group was lower than that in the reference group, and the incidence of pharyngeal mucosal injury in the research group was lower than that in the reference group $(\mathrm{P}<0.05)$. The pain score of the study group was lower than that of the reference group at $8 \mathrm{~h}, 12 \mathrm{~h}$ and $24 \mathrm{~h}$ after replacement, and the incidence of hoarseness, swallowing discomfort and dry oropharynx was lower than that of the reference group $(\mathrm{P}<0.05)$. Conclusion: The application of modified gastric tube placement and Rehabilitation New Liquid Spray intervention in patients with indwelling gastric tube can effectively reduce the nasopharyngeal injury and improve the pain of catheterization.
\end{abstract}

Keywords: Modified gastric tube placement; Indwelling gastric tube; New liquid spray

Publication date: November 2021; Online publication: November 30, 2021

\section{Introduction}

Currently, nasal placement of gastric tube is a commonly used technology in clinical nursing. The gastric tube needs to be placed from the patient's nasal cavity, and the gastric tube must be able to pass through the pharynx normally and smoothly, and slide into the esophagus along the posterior wall of the pharynx. In the past, the traditional methods of gastric tube placement usually required patients to cooperate with swallowing ${ }^{[1]}$. The failure rate of gastric tube placement was relatively high, about $1.3-2.4 \%$, especially for special groups such as dysphagia, anesthesia and children. In addition, if the gastric tube placement was misplaced, it would also lead to respiratory complications and death. Therefore, improving gastric tube implantation in time to effectively improve the success rate of gastric tube implantation is a major hot issue in clinical nursing research. The traditional supine gastric tube placement method is a commonly used method in clinic, but this method will cause some discomfort to patients. Since it belongs to invasive operation technology, it will physically stimulate and pull the patient's throat during gastric tube placement, resulting in foreign body sensation in the throat, and may even lead to a certain degree of pharyngeal congestion, mucosal damage, choking and other reactions, resulting in dry throat, hoarseness, nausea and other symptoms, which affect the treatment effect ${ }^{[2]}$. Therefore, it is necessary to explore and devise an effective gastric tube placement method, so as to shorten the operation practice, improve the success rate of gastric tube placement, and alleviate the pain arising from gastric tube placement. In view of this, the nursing effect of improved gastric tube placement combined with new liquid spray was explored. 


\section{Materials and methods}

\subsection{Materials}

Eighty-six patients who received gastric tube indwelling in our hospital from January 2020 to May 2021 were randomly selected as the research subjects. The inclusion criteria are as follows: (1) All of them were over 18 years old; (2) No respiratory symptoms; (3) Oral and nasal mucosa examination were normal; (3) Conscious, able to communicate normally and cooperate with various treatments; (5) The study was approved by the medical ethics committee of our college. The patients voluntarily participated in the study and voluntarily signed the informed consent forms. The exclusion criteria are as follows: (1) Combined with severe liver and kidney insufficiency and autoimmune diseases; (2) Non-infectious inflammation and systemic inflammatory reaction caused by bacteria; (3) Gastric tube cannot be placed due to nasopharynx lesions; (4) Suffering from a disease in which the mouth cannot open; (5) Severe oral ulcer that interferes with the evaluation; (6) People allergic to lidocaine mucilage and Rehabilitation New Liquid; (7) People who was mentally abnormal and unable to cooperate, etc.

The group was divided into reference group and research group, with 43 cases in each group ${ }^{[3]}$. The ratio of men to women in the reference group was 23:20. The age range was 31-63 years, with an average of $45.13 \pm 1.25$ years old. The ratio of men to women in the research group was $24: 19$. The age range was 32-64 years, with an average of $45.24 \pm 1.31$ years. There was no significant difference in the patient's data calculated by statistical software $(\mathrm{P}>0.05)$.

\subsection{Methods}

The reference group was treated with improved body position and gastric tube placement. The methods included right gastric tube placement, neck bending lateral compression, reverse Sellick method, rotary placement and sitting gastric tube placement. The patients took the right lying position while gastric tube placement was ongoing, with the head, neck and trunk remain in the right lying position on the same plane. The gastric tube was inserted $10-15 \mathrm{~cm}$ through the nose. The lateral lying angle of the patient's body was increased in case of increasing resistance to make the tongue move forward under the action of gravity. The negative pressure drainage bag was replaced every day; the drainage was kept unobstructed; the patients were requested not to rotate their heads and swallow frequently, as well as not to pull out the tube by themselves; psychological counseling was conducted regularly ${ }^{[4]}$.

On the basis of the treatment used in research group, Rehabilitation New Liquid Spray intervention was also adopted. First, when the tube was placed, the lidocaine mucilage was applied for anesthesia before the gastric tube insertion. After successful catheterization, the patients were given spray nursing intervention with Rehabilitation New Liquid. The $20 \mathrm{ml}$ Rehabilitation New Liquid was injected into the throat atomizer accurately, and then the patient was given oral and nasal spray with the help of a nebulizer. The dosage scheme is as follows: 4 times a day, 3-4 sprays each time, $0.5 \mathrm{ml}$ each spray, performed at night. During the process, patients were instructed to use inspiratory and rapid swallowing so that the liquid can quickly enter the nasal cavity and throat of the patient, with a spray intervals of 2 hours ${ }^{[5]}$.

\subsection{Observation indexes}

The evaluation criteria for nasal mucosal injury are as follows:

(1) Grade 0 injury: if the patient's nasopharyngeal mucosa is normal;

(2) Grade I injury: if the patient's nasal mucosa has mild pain or erythema;

(3) Grade II injury: if the patient's nasal mucosa shows moderate erythema and edema;

(4) Grade III injury: if nasal mucosa shows mild ulcer; and

(5) Grade IV injury: if the patient's nasal mucosa has moderate ulcer or necrosis. 
The total incidence formula is given below:

Total incidence rate $=($ grade $\mathrm{I}+$ grade II + grade III + grade IV $) /$ total number of cases $\times 100 \%$

The pain score scale (VAS) was used to evaluate the pain of the two groups at the time of gastric tube indwelling, $8 \mathrm{~h}, 12 \mathrm{~h}$, and $24 \mathrm{~h}$ after indwelling. The total score was 10 points. The higher the score is, the more serious the pain is. The occurrence of hoarseness, dysphagia and dry oropharynx after gastric tube indwelling in the two groups were observed and recorded.

\subsection{Statistical analysis}

The data are mainly processed and analyzed by SPSS version 23.0 software, analyzed using $t$ test and Chisquared test, and expressed as either mean \pm standard deviation (SD) or count (percentage). The processing and analysis result with $\mathrm{P}<0.05$ indicates statistically significant difference.

\section{Results}

\subsection{Observation of the nasal mucosa injury}

According to the evaluation statistics, incidence of nasal mucosal injury of research group was lower than that of reference group $(\mathrm{P}<0.05)$, as shown in Table $\mathbf{1}$.

\subsection{Observation of the pharyngeal mucosa injury}

Based on observation of the pharyngeal mucosa injury, incidence of pharyngeal mucosal injury of the research group was lower than that of the reference group $(\mathrm{P}<0.05)$, as shown in Table 2.

\subsection{Pain score}

According to the statistics of the scoring scale, there was no significant difference in pain score between the two groups when indwelling $(\mathrm{P}>0.05)$. However, at $8 \mathrm{~h}, 12 \mathrm{~h}$, and $24 \mathrm{~h}$ after indwelling, the pain scores of the research group were lower than those of the reference group $(\mathrm{P}<0.05)$, as shown in Table 3.

\subsection{Observation of the incidence of adverse events after indwelling gastric tube}

According to the observation statistics, incidence of hoarseness, swallowing discomfort and dry oropharynx of research group was lower than that of reference group $(\mathrm{P}<0.05)$, as shown in Table 4.

Table 1. Statistics of the incidence of nasal mucosal injury

\begin{tabular}{lccccccc}
\hline Group & N (example) & Grade 0 & Grade I & Grade II & Grade III & Grade IV & Total incidence rate \\
\hline Reference group & 43 & 30 & 7 & 3 & 2 & 1 & 30.23 \\
Research Group & 43 & 38 & 3 & 1 & 1 & 0 & 11.62 \\
Chi-squared & & & & & & & 4.4967 \\
P & & & & & & & 0.0339 \\
\hline
\end{tabular}

Table 2. Statistics of the incidence of pharyngeal mucosal injury

\begin{tabular}{lccccc}
\hline Group & N (example) & Grade 0 & Grade I & Grade II & Total incidence rate \\
\hline Reference group & 43 & 31 & 8 & 4 & 27.90 \\
Research Group & 43 & 39 & 3 & 1 & 9.30 \\
Chi-squared & & & & & 4.9143 \\
P & & & & & 0.0266 \\
\hline
\end{tabular}


Table 3. Statistical pain score

\begin{tabular}{lccccc}
\hline Group & N (example) & When indwelling & $\begin{array}{c}\mathbf{8} \text { h after } \\
\text { indwelling }\end{array}$ & $\begin{array}{c}\mathbf{1 2} \text { h after } \\
\text { indwelling }\end{array}$ & $\begin{array}{c}\mathbf{2 4} \text { h after } \\
\text { indwelling }\end{array}$ \\
\hline Reference group & 43 & $5.38 \pm 0.75$ & $4.85 \pm 0.62$ & $3.74 \pm 0.55$ & $3.25 \pm 0.46$ \\
Research group & 43 & $5.41 \pm 0.76$ & $3.91 \pm 0.51$ & $3.21 \pm 0.43$ & $2.87 \pm 0.28$ \\
t & & 0.1842 & 7.6780 & 4.9781 & 4.6272 \\
P & & 0.8543 & 0.0000 & 0.0000 & 0.0000 \\
\hline
\end{tabular}

Data are expressed as mean $\pm \mathrm{SD}$.

Table 4. Statistics of the incidence of unfavorable condition

\begin{tabular}{lcccc}
\hline Group & N (example) & Hoarseness & Swallowing discomfort & Dry oropharynx \\
\hline Reference group & 43 & $8(18.60)$ & $9(20.93)$ & $10(23.25)$ \\
Research Group & 43 & $2(4.65)$ & $2(4.65)$ & $3(6.97)$ \\
Chi-squared & & 4.0737 & 5.1079 & 4.4405 \\
P & & 0.0435 & 0.0238 & 0.0350 \\
\hline
\end{tabular}

Data are expressed as count (percentage).

\section{Discussion}

Presently, nasal placement of gastric tube is a commonly used technique in clinical nursing. In the past, the gastric tube placement method employing traditional supine position was often used. However, after the placement of gastric tube and indwelling gastric tube, patients will generally show some discomfort symptoms, and this may lead to repeated intubation failure or unplanned extubation due to unbearable pain, ultimately affecting the therapeutic effect. Therefore, it is necessary to modify the gastric tube placement method to shorten the operation time and improve the comfort of tube placement ${ }^{[6]}$.

The clinical study found that the improved right lateral gastric tube placement method is significantly advantageous over the gastric tube placement method using traditional supine position. At the same time, during the operation, the use of lidocaine mucilage for pharyngeal surface anesthesia, and rehabilitation new liquid pharyngeal spray after the successful catheterization can effectively improve the uncomfortable symptoms arising from gastric tube placement, and reduce the related complications that may be caused by indwelling gastric tube, so as to alleviate the pain due to indwelling gastric tube and improve comfort. This, it is of significance to improve the pharyngeal symptoms and compliance of patients with indwelling gastric tube.

In conclusion, the application of modified gastric tube placement and Rehabilitation New Liquid Spray intervention in patients with indwelling gastric tube can effectively reduce the nasopharyngeal injury and alleviate the pain due to catheterization ${ }^{[7-9]}$.

\section{Disclosure statement}

The author declares no conflict of interest.

\section{References}

[1] Ma LY, Zhao M, Gao MJ, et al., 2019, Application of Modified Adhesive Tape Fixation in Indwelling Gastric Tube in Elderly Patients. Journal of Clinical Medical Literature, 6(22): 42+45.

[2] Zhu LJ, Guo HT, 2020, Application of Modified Gastric Tube Placement based on Anatomical 
Structure in Patients with Stroke Undergoing Tracheotomy. Nursing Journal of Chinese People's Liberation Army, 037(005): 90-92.

[3] Xiao HY, Wang Y, Chen XF, et al., 2019, Effect Observation of Lower Esophageal Gastric Tube Implantation on Postoperative Enteral Nutrition Support in Patients with Laryngeal Cancer. Smart Healthcare, 000(005): 129-130.

[4] Weng XM, Wei XJ, Li P, 2020, Effect Study of Modified Indwelling Gastric Tube Method on Indwelling Gastric Tube in Stroke Patients with Swallowing Dysfunction. Contemporary Medicine Forum, 18(04): 113-114.

[5] Han HM, Liu J, 2017, Application Study of Combined Gastric Tube Placement in Endotracheal Intubation under General Anesthesia. Modern Nurse, 000(006): 130-131.

[6] Kong M, Zhou XM, Kong, et al., 2018, Application Research of Cold Acid Stimulation of Throat with Plum Popsicle Combined with Throat Touch in Nasogastric Tube Implantation. Nursing Practice and Research, 15(02): 41-43.

[7] Zhang JJ, Li JD, 2020, Application of Rehabilitation New Liquid Plus Lidocaine Mucilage Mixture in Improving the Comfort of Pharynx in Patients with Gastrointestinal Decompression. Clinical Research and Practice, 5(17): 159-161.

[8] Wei JH, Zou XL, 2018, Effect Observation of Rehabilitation New Liquid in Improving the Pharyngeal Comfort in Patients with Gastrointestinal Decompression. Nursing of Integrated Traditional Chinese and Western Medicine (Chinese and English), 2018(7): 114-117.

[9] Liu P, 2020, Improved Indwelling Gastric Tube Combined with Rehabilitation Nursing of Traditional Chinese Medicine to Improve the Nutritional Status of Patients with Swallowing Dysfunction in the Early Stage of Cerebral Infarction. Heilongjiang Science, 181(18): 56-57. 